\title{
25 Research Soure \\ Effects of Robot-Aided Rehabilitation on Improving Ankle and Balance Performance of Stroke Survivors: A Randomized Controlled Trial
}

\section{Xiaoxue Zhai}

Tsinghua University School of Medicine https://orcid.org/0000-0001-5650-4919

Qiong Wu

Tsinghua University School of Medicine

Quan Xu

Tsinghua University School of Medicine

Xin Li

Tsinghua University School of Medicine

Yanlin Zhang

Tsinghua University School of Medicine

Senchao Fan

Tsinghua University School of Medicine

\section{Li-Qun Zhang}

University of Maryland Baltimore

Yu Pan ( $\nabla$ panyu@btch.edu.cn )

Department of Rehabilitation, Beijing Tsinghua Changgung Hospital https://orcid.org/0000-00025703-5322

\section{Research}

Keywords: Stroke, Ankle, Spasticity, Balance, Rehabilitation

Posted Date: July 24th, 2020

DOI: https://doi.org/10.21203/rs.3.rs-30969/v2

License: (c) (1) This work is licensed under a Creative Commons Attribution 4.0 International License. Read Full License 


\section{Abstract}

Background: Stroke survivors often experience abnormal posture control, which affects balance and locomotion. The ankle strategy is important in maintaining static balance. Prolonged spasticity may result in biomechanical changes at the ankle joint, which may cause balance disorders. The intelligent stretching device may decrease the stiffness of the ankle and improve balance. The purpose of this study was to investigate the effects of robot-aided ankle rehabilitation of stroke survivors with ankle spasticity and the correlations between biomechanical properties and balance in these patients.

Methods: Twenty inpatients post stroke with ankle spasticity performed 20 minutes of stretching treatment for 2 weeks. The study group used a rehabilitation robot to stretch the spastic ankle plantar flexors under intelligent control and the control group received manual stretching. Outcome measures included biomechanical, clinical evaluations and Pro-Kin balance test.

Results: After training, significant improvements were found in both groups in the active range of motion, muscle strength, Berg Balance Scale, Fugl-Meyer Motor Assessment of Lower Extremity, Postural Assessment Scale for Stroke Patients, 6-minute walk test, and Modified Barthel Index $(P<0.05)$; significant decreases were found in the study group in dorsiflexion stiffness, Modified Ashworth Scale, trajectory lengths, elliptical trajectory, standard deviation medial/lateral, average speed forward/backward with eyes closed, and standard deviation forward/backward with eyes open $(P=0.001, P=0.037, P=0.028$, $P=0.019, P=0.016, P=0.001$, and $P=0.033$, respectively); dorsiflexion stiffness was positively correlated with the Pro-Kin balance test outcomes: ellipse area, trajectory length, average speed forward/backward, average speed medial/lateral with eyes open $(=0.352, \mathrm{P}=0.026 ;=0.522, \mathrm{P}=0.001 ;=0.045, \mathrm{P}=0.004$; $=0.433, P=0.005$, respectively); dorsiflexion stiffness was correlated with the Modified Ashworth Scale ( $=0.265, P=0.041)$; the study group improved significantly more than the control group in the activities of daily living after training $(P=0.017)$.

Conclusions: The results suggested that robot-aided ankle rehabilitation had a positive effect on the biomechanical properties of the spastic ankle, and it may be feasible to improve balance post-stroke. Ankle dorsiflexion stiffness affected balance poststroke significantly; it may be a sensitive indicator for evaluating balance.

Trial registration: www.chictr.org.cn ChiCTR1900022128. Registered 21 February 2020. Retrospectively registered.

\section{Background}

Stroke survivors often experience spasticity, joint contractures, muscle weakness, and motor impairments that impede ambulation and balance and contribute directly to disabilities [1, 2]. Foot drop is a common ankle impairment that affects locomotion post-stroke [3]. Deterioration in ankle joint motion can result in clumsy gait patterns with excessive energy cost [4] and balance disorders. Previous studies have found that foot drop can be caused by impaired voluntary control, weakness of the dorsiflexor muscles or 
spastic plantar flexors, which can limit foot clearance during the swing phase as well as reduce loading ability during the mid-stance of gait [5].

Lack of mobilization and prolonged spasticity may be accompanied by structural changes in the muscle fibers and connective tissue, which may result in biomechanical changes at the ankle, including reduced range of motion (ROM), increased resistance and stiffness, and muscular imbalance, leading to a clinical contracture [6-9]. Recently, an intelligent stretching device was developed to decrease ankle stiffness of patients who were neurologically impaired due to stroke, spinal cord injury, multiple sclerosis, or cerebral palsy. Significant improvements were found in the ROM, maximum voluntary contraction, ankle stiffness, and comfortable walking speed [10-15].

As a crucial part of posture control, ankle strategy is important during daily ambulation and functional activities [16]. However, the correlations between the local biomechanical properties of the ankle and balance are unclear. Therefore, this study aimed to perform repeated intelligent stretching of the ankle with spasticity in patients with hemiplegia after stroke, observing the biomechanical changes in the ankle after training, and assessing static balance quantitatively using the Pro-Kin device [17], based on the assessment of postural sway using the force platform from movements of the center of pressure (CoP), and to further explore the correlations between the Pro-Kin balance test outcomes and ankle stiffness quantitatively, to determine the effect of ankle stiffness on balance.

\section{Methods}

\section{Participants}

This single-blinded, randomized controlled trial was conducted at the Beijing Tsinghua Chang Gung Hospital in China. Inpatients with subacute stroke in rehabilitation department of hospital were recruited between March 2019 and August 2019. The inclusion criteria were: (1) ages between 18 and 75 years; (2) first-ever stroke with less than 6 months duration of spasticity of the affected ankle (Modified Ashworth Scale, MAS: 1 2); (3) medically stable; (4) ability to stand independently without aids for at least 1 minute; and (5) ability to perform the experimental treatment independently. Exclusion criteria were communication problems, dementia based on clinical diagnosis, comorbidities affecting motor performance such as orthopedic, arthritic, inflammatory conditions that could influence balance, and limited ankle movement.

\section{Sample size calculation}

Sample size calculation was conducted using G*Power 3.1.7 (http://www.gpower.hhu. de/). The effect size was estimated using our pilot data that $\mathrm{N}$ patients per study group (study group vs control group: $0.61 \pm 0.21$ vs $0.31 \pm 0.27$ ) would be able to reveal a large effect size of Cohen's $d=1.24$, at a power of 0.8 and an a level of 0.05 assuming a non-directional hypothesis. Thus, in current study, a large effect size 
$\mathrm{f}=0.4$ was assumed in ANOVA model, with an a value of 0.05 , power of 0.8 and an attrition rate of $10 \%$, the minimum required sample size was estimated to be 18 participants for this study.

\section{Experimental design}

A physical therapist who was not involved in this study used a computer to generate a random number table, and designated the random numbers as the study group or the control group according to odd or even numbers. Patients were randomized into the study group and the control group with 1:1 ratio. The pre-designed random number list was put into the sequentially coded, sealed and opaque envelope. When a rehabilitation physician not involved in the study was determined that the patients met the conditions, the envelope was opened in order and the patients were enrolled sequentially and assigned to either the study group or the control group. The appointed therapist in charge of the assessment was blinded to the mode of training received by the patients throughout the study period. Patients in the study group had 10 sessions (five times a week over 2 weeks). Each session comprised 20 minutes training by stretching the ankles with spasticity under intelligent control. Training for the control group involved manual stretching of the plantar flexors (five times a week over 2 weeks, 20 minutes/session). During the 2-week period, both groups continued active movement exercises for ankle mobility and strength.

\section{Devices}

An ankle rehabilitation robot (Beijing LTK Science and Technology Co., Ltd) was used for intervention and outcome evaluations [18]. The leg of the subject was strapped to a leg support with the knee at $10^{\circ}$ flexion and the foot was strapped onto a footplate with ankle dorsiflexion (DF) at $0^{\circ}$. The footplate was fixed to the motor shaft, and a torque sensor was aligned with the motor shaft to measure the ankle joint torque (Fig. 1). The ankle rehabilitation robot was driven by a servomotor controlled by a digital signal processor [10]. Briefly, the stretching velocity was inversely proportional to the joint resistance torque. Typical values were $30 \%$ s peak stretching velocity, 10 to $40 \mathrm{Nm}$ peak resistance torque in dorsiflexion, 5 to $10 \mathrm{Nm}$ peak resistance torque in plantarflexion, and a 5-s holding period at extreme dorsiflexion [11]. An experienced physical therapist adjusted the peak resistance torque for each session based on manual stretching and feedback from the subject during the stretching.

\section{Assessments}

Subjects were tested before and after the training period by the appointed blinded physical therapist. Biomechanical, clinical evaluations and the Pro-Kin balance test (PK252, TecnoBody, Italy) were conducted. All assessment sessions were performed at the same time of day and in the same order. 


\section{Biomechanical evaluations}

Evaluations included the passive and active ranges of motion (PROM, AROM), dorsiflexor and plantarflexor muscle strength, and DF and plantarflexion (PF) stiffness. ROM and muscle strength were measured using the HogganMicroFET3 portable device (Hoggan Health Industries, Inc. Salt Lake City, USA). The measuring range of ROM was $0-180^{\circ}$ with an accuracy of $1^{\circ}$; the measuring range of muscle strength was 3.6-890 N with an accuracy of $0.01 \mathrm{~N}$ (Fig. 2). The stiffness measured in DF or PF passive movement was assessed as $\mathrm{K}=\Delta \mathrm{T} / \Delta \theta$, where $\mathrm{K}(\mathrm{Nm} / \mathrm{deg})$ was the quasi-static stiffness and $\Delta \mathrm{T}$ was the passive torque increment during a certain amount of ankle angular movement $(\Delta \theta)$. As $\Delta \theta$ becomes infinitely small, the quasi-static stiffness approaches the slope of a tangential line of the torque-angle curve at a specific ankle position $[10][19,20]$. The peak stretching velocity in this study was set at $5 \%$ s to avoid inducing reflex responses [21]. Quasi-static stiffness of the ankle plantar flexor (stiffness measured in DF direction movement, DF stiffness) was evaluated at $10^{\circ}$ of DF and that of the ankle dorsiflexor (stiffness measured in PF direction movement, PF stiffness) at $30^{\circ}$ of PF. Three trials were conducted and the averages were taken to be AROM, PROM, muscle strength, and stiffness $(K)$.

\section{Clinical evaluations}

Each subject completed the following functional assessments during clinical evaluation sessions. MAS (0-4 points) [22] was used to measure the calf muscle hypertonia. Fugl-Meyer Motor Assessment of Lower Extremity (FM-LE) [23] (0-34 points) was used to evaluate the sensorimotor function of the lower limbs. The Berg Balance Scale (BBS) [24] (0-56 points) was used to evaluate the balance function. The 6-minute walk test (6MWT) [25] was used to determine walking ability. The Postural Assessment Scale for Stroke Patients (PASS) [26] (0-36 points) was used to evaluate the ability to control posture. The Modified Barthel Index (MBI) [27] (0-100 points) was used to measure the activities of daily living (ADL).

\section{Pro-Kin balance evaluations}

This study also used the Pro-Kin system (PK252, TecnoBody, Italy) to assess balance function, which was based on the assessment of postural sway using the force platform from movements of the center of pressure (CoP) [28-30] (Fig. 3). Subjects stood on the platform comfortably, looking straight ahead at a screen and keeping arms at their sides during the stances, with eyes focused on a stationary target. Each participant performed two standing tests, with opened eyes (OE) and closed eyes (CE), each test lasting $30 \mathrm{~s}$, using a sampling frequency of $20 \mathrm{~Hz}$. Postural sway was determined using six different outcome variables: trajectory lengths (measured in $\mathrm{mm}$ ), elliptical trajectory (measured in $\mathrm{mm}^{2}$ ), standard deviation medial/lateral (M/L SD), standard deviation forward/backward (F/B SD), average speed 
medial/lateral (M/L AS measured in $\mathrm{m} / \mathrm{s}$ ), and average speed forward/backward ( $\mathrm{F} / \mathrm{B}$ AS measured in $\mathrm{m} / \mathrm{s}$ ). The trajectory lengths referred to the length of the trajectory of the pressure center; the greater the length, the poorer the balance function the patient displayed. Elliptical trajectory referred to the area surrounded by the trajectory of the center of the body pressure; the larger the area, the poorer the balance function the patient demonstrated. An increase in CoP in either the forward/backward or medial/lateral direction was indicative of postural disturbance, including SD and AS. Smaller values of the six parameters indicated the patient had a better balance function [31].

\section{Statistical analysis}

Differences in general characteristics of the subjects between the two groups were assessed using the Mann-Whitney U test or Fisher's exact test. For all outcome variables, the group mean and standard deviation at pre- and post-training were calculated. The values were compared between the groups using the two-way repeated ANOVA. Post-hoc paired sample t-test with Bonferroni correction within the study and control groups was performed. We used Pearson's coefficient to examine the correlation between stiffness $(K)$ and the Pro-Kin system results, and the Kendall rank correlation coefficient $(\tau)$ for the correlation between MAS and K [32]. A significance level of $p \leq 0.05$ was set for two-way repeated ANOVA models and correlation tests. All statistical tests were analyzed using IBM SPSS Statistics for Windows, version 21.0. (IBM Corporation, Armonk, NY, USA).

\section{Results}

\section{The flow of the trial and baseline characteristics of patients}

From March 2019 to August 2019, all inpatients in the rehabilitation department were screened. Of these, 43 patients post stroke with ankle spasticity were eligible for evaluation. Among these patients, 20 patients did not meet the inclusion criteria and 3 patients declined to participate in this study (see Fig. 4 for more details). A total of 20 patients were recruited to the study, including 10 patients randomized to the study group, and 10 patients randomized to the control group. All enrolled participants completed the full 2-week treatment, and there were no dropouts or adverse events. There were no significant differences in age, duration post stroke, sex, stroke type, side of lesion, height, weight, or BMI (Body Mass Index) between the two groups (Table 1). 


\begin{tabular}{|c|c|c|c|}
\hline Parameters & Study group $(n=10)$ & Control group $(\mathrm{n}=10)$ & P Value \\
\hline Age\year & $9 / 1$ & $9 / 1$ & 0.613 \\
\hline duration post stroke & $10 / 0$ & $10 / 0$ & 0.841 \\
\hline $\operatorname{Sex}(M / F)$ & $7 / 3$ & $7 / 3$ & \\
\hline \multicolumn{4}{|c|}{ Cerebral infarction/cerebral hemorrhage (case) } \\
\hline \multicolumn{4}{|c|}{ Side of lesion (left/right, case) } \\
\hline Height & & & 0.573 \\
\hline Weight ( & & & 0.237 \\
\hline BMI ( & & & 0.158 \\
\hline
\end{tabular}

P-values indicate the results of Mann-Whitney U Test for age and duration post stroke, and of Fisher's exact test for all the other variables.

Values are mean \pm standard deviation, or number. BMI, Body Mass Index; M, male; F, female

\section{Biomechanical evaluations: PROM, AROM, muscle strength and joint stiffness}

Before training there were no significant differences in PROM, AROM, muscle strength, or DF and PF stiffness between the two groups. The DF AROM, PF AROM, DF muscle strength, and PF muscle strength increased significantly after the 2-week training period in the control group ( $\mathrm{P}=0.032 ; \mathrm{P}=0.001 ; \mathrm{P}=0.011 ; \mathrm{P}<0.0001$, respectively). The four parameters of biomechanical properties showed similar changes in the control group as in the treatment group $(\mathrm{P}=0.001 ; \mathrm{P}=0.002 ; \mathrm{P}=0.007 ; \mathrm{P}=0.001$, respectively $)$. In addition, significant decreases in DF stiffness were found for subjects in the study group $(\mathrm{P}=0.001)$ but not in the control group. No significant differences in biomechanics were found between the two groups after training (P>0.05) (Table 2, Fig. 5).

Table 2. Biomechanical Properties at pre- and post-treatment between two groups 


\begin{tabular}{|c|c|c|c|c|}
\hline \multirow[b]{2}{*}{ Variable } & \multicolumn{2}{|c|}{ Study group } & \multicolumn{2}{|c|}{ Control group } \\
\hline & Pre-training & Post-training & Pre-training & Post-training \\
\hline \multicolumn{5}{|l|}{ DF AROM $\left({ }^{\circ}\right)$} \\
\hline \multicolumn{5}{|l|}{$\operatorname{DF}$ PROM $\left(^{\circ}\right)$} \\
\hline \multicolumn{5}{|l|}{$\operatorname{PF} \operatorname{AROM}\left({ }^{\circ}\right)$} \\
\hline \multicolumn{5}{|l|}{$\operatorname{PF} \operatorname{PROM}\left({ }^{\circ}\right)$} \\
\hline \multicolumn{5}{|l|}{ DF Strength (N) } \\
\hline \multicolumn{5}{|l|}{ PF Strength (N) } \\
\hline \multicolumn{5}{|l|}{ DF Stiffness (Nm/deg) } \\
\hline PF Stiffness (Nm/deg) & & & & \\
\hline
\end{tabular}

Values are mean \pm standard deviation. $* \mathrm{p}<.05$ for Pre vs Post, ${ }^{\S} \mathrm{p}<.05$ for Study group vs Control group Post-training. The change of biomechanical properties over time within each group was evaluated using Post-hoc paired sample t-test with Bonferroni correction. The values were compared between the groups using the two-way repeated ANOVA. DF or PF stiffness is stiffness measured in dorsiflexion or plantarflexion passive movement.

\section{Clinical evaluations}

There was no significant difference in MAS, FM-LE, BBS, PASS, 6MWT, or MBI before training between the two groups. The FM-LE, BBS, PASS, 6MWT, and MBI increased significantly after the 2 -week training period in the control group $(\mathrm{P}=0.006 ; \mathrm{P}=0.004$; $\mathrm{P}=0.002 ; \mathrm{P}=0.018 ; \mathrm{P}=0.029$, respectively). We also found significant improvement in $\mathrm{FM}$ LE, BBS, PASS, 6MWT, and MBI in the study group $(\mathrm{P}=0.001 ; \mathrm{P}=0.037 ; \mathrm{P}=0.009 ; \mathrm{P}=0.028$; $\mathrm{P}=0.001$, respectively). In addition, significant decreases were found in MAS for subjects in the study group $(\mathrm{P}=0.037)$ but not in the control group. Subjects in the study group improved significantly more than those in the control group in the activities of daily living (ADL) by MBI after the 2-week training ( $\mathrm{P}=0.017$ ) (Table 3, Fig. 6, 7).

Table 3. Clinical evaluations at pre- and post-treatment between two groups

\begin{tabular}{|c|c|c|c|c|}
\hline \multirow[b]{2}{*}{ Variable } & \multicolumn{2}{|c|}{ Study group } & \multicolumn{2}{|c|}{ Control group } \\
\hline & Pre-training & Post-training & Pre-training & Post-training \\
\hline \multicolumn{5}{|l|}{ MAS } \\
\hline \multicolumn{5}{|l|}{ FM-LE } \\
\hline \multicolumn{5}{|l|}{ BBS } \\
\hline \multicolumn{5}{|l|}{ PASS } \\
\hline \multicolumn{5}{|l|}{$6 \mathrm{MWT}$} \\
\hline MBI & & & & \\
\hline
\end{tabular}


Values are mean \pm standard deviation. $* \mathrm{p}<.05$ for Pre vs Post, ${ }^{\S} \mathrm{p}<.05$ for Study group vs Control group Post-training. The change of Clinical evaluations over time within each group was evaluated using Post-hoc paired sample t-test with Bonferroni correction. The values were compared between the groups using the two-way repeated ANOVA. MAS Modified Ashworth Scale, FM-LE Fugl-Meyer Motor Assessment of Lower Extremity, BBS Berg Balance Scale, PASS Postural Assessment Scale for Stroke Patients, 6 MWT 6-minute walk test, MBI Modified Barthel Index.

\section{Pro-Kin balance test outcomes}

There was no significant difference between the two groups in ellipse area, trajectory length, F/B SD, L/M SD, F/B AS, or L/M AS with opened and closed eyes before training. The ellipse area, trajectory length, L/M SD, and F/B AS with closed eyes and F/B SD with opened eyes decreased significantly after the 2-week training period in the study group ( $\mathrm{P}=0.028 ; \mathrm{P}=0.019 ; \mathrm{P}=0.016 ; \mathrm{P}=0.001 ; \mathrm{P}=0.033$, respectively). We found no significant change in the control group. No significant difference in the Pro-Kin balance test outcomes was found between the two groups after training $(\mathrm{P}>0.05)$ (Table 4). Examples of the trajectory of the CoP of two groups are shown in Fig. 8, 9. It can be noticed that the participant S09 attained a better balance function after training than participant C07 significantly.

Table 4. Pro-Kin balance test outcome at pre- and post-training between two groups

\begin{tabular}{|c|c|c|c|c|}
\hline \multirow[t]{2}{*}{ Variable } & \multicolumn{2}{|c|}{ Study group } & \multicolumn{2}{|c|}{ Control group } \\
\hline & Pre-training & Post-training & Pre-training & Post-training \\
\hline \multicolumn{5}{|l|}{ Eyes Closed } \\
\hline \multicolumn{5}{|l|}{ Ellipse Area } \\
\hline \multicolumn{5}{|l|}{ Trajectory Length } \\
\hline \multicolumn{5}{|l|}{ F/B SD } \\
\hline L/M SD & 10.10 & & & \\
\hline \multicolumn{5}{|l|}{$\mathrm{F} / \mathrm{B}$ AS mm/sec } \\
\hline \multicolumn{5}{|l|}{$\mathrm{L} / \mathrm{M}$ AS mm/sec } \\
\hline \multicolumn{5}{|l|}{ Eyes Open } \\
\hline \multicolumn{5}{|l|}{ Ellipse Area } \\
\hline \multicolumn{5}{|l|}{ Trajectory Length } \\
\hline \multicolumn{5}{|l|}{$\mathrm{F} / \mathrm{B} \mathrm{SD}$} \\
\hline \multicolumn{5}{|l|}{$\mathrm{L} / \mathrm{M} \mathrm{SD}$} \\
\hline \multicolumn{5}{|l|}{$\mathrm{F} / \mathrm{B}$ AS mm/sec } \\
\hline $\mathrm{L} / \mathrm{M}$ AS mm/sec & & & & \\
\hline
\end{tabular}


Values are mean \pm standard deviation. $* \mathrm{p}<.05$ for Pre vs Post, ${ }^{\S} \mathrm{p}<.05$ for Study group vs Control group Post-training. The change of Pro-Kin balance test outcome over time within each group was evaluated using Post-hoc paired sample t-test with Bonferroni correction. The values were compared between the groups using the two-way repeated ANOVA. AS Average speed, Forward/Backward F/B, Medial/Lateral direction M/L.

\section{Correlations between stiffness of the ankle and the balance function}

The DF stiffness was significantly correlated with the outcomes of the Pro-kin balance test with opened eyes, including the ellipse area, trajectory length, F/B AS, M/L AS ( $=0.352$, $\mathrm{P}=0.026 ;=0.522, \mathrm{P}=0.001 ;=0.045, \mathrm{P}=0.004 ;=0.433, \mathrm{P}=0.005$, respectively). The $\mathrm{DF}$ stiffness was also significantly correlated with MAS ( $=0.265, \mathrm{P}=0.041)$ (Table 5).

Table 5 Correlations Between stiffness and Pro-Kin balance test outcome when open eyes

\begin{tabular}{|c|c|c|c|c|}
\hline \multicolumn{2}{|c|}{ DF stiffness } & $\mathrm{P}$ value & PF stiffness & $\mathrm{P}$ value \\
\hline MAS & 0.265 & $0.041^{*}$ & & 0.344 \\
\hline Ellipse Area & 0.352 & $0.026^{*}$ & & 0.179 \\
\hline Trajectory Length & 0.522 & $0.001 *$ & & 0.050 \\
\hline F/B SD & 0.285 & 0.075 & & 0.331 \\
\hline $\mathrm{M} / \mathrm{L} \mathrm{SD}$ & 0.277 & 0.084 & & 0.101 \\
\hline F/B AS & 0.450 & $0.004^{*}$ & & 0.077 \\
\hline $\mathrm{M} / \mathrm{L}$ AS & 0.433 & $0.005 *$ & & 0.116 \\
\hline
\end{tabular}

Pearson's coefficient $(\gamma)$ was used to estimate the correlation between stiffness $(\mathrm{K})$ and the Pro-Kin system results, and the Kendall rank correlation coefficient $(\tau)$ was used to estimate the correlation between stiffness $(K)$ and MAS. Values are $\gamma$ or $\tau$. *significance level of $p \leq 0.05$ was set for correlation tests.

\section{Discussion}

The main findings of our randomized controlled pilot study were that both conventional manual and robotic intelligent stretching of spastic ankles could improve the biomechanical properties of the ankle joints and motor function. For the study group, the spasticity of the ankle decreased more as evaluated by DF stiffness and MAS, and the balance function improved more as evaluated by the Pro-Kin balance test compared with the control group. This study found that robot-aided rehabilitation of the ankle could invoke more benefits in joints with impaired biomechanical properties than the methods used in the control group by decreasing stiffness of the spastic ankle to improve balance and activities of daily living. The stiffness of dorsiflexion as a crucial biomechanical property of the local ankle joint influenced the overall balance in stroke deeply; it may be a sensitive indicator for evaluating the ability to balance. 
All subjects completed the intervention successfully, and there were no adverse effects reported for this research.

\section{Biomechanical properties of the spastic ankle}

Several studies have reported the positive effects of stretching subjects with joint spasticity and/or contracture by decreasing muscle tone, improving soft-tissue extensibility, and increasing ankle ROM [3339]. Manual stretching by moving the spastic ankle through its ROM requires labor-intensive efforts, and the outcome is dependent on the experience of the therapist, which limits its availability. Thus, external devices [40,41] and robotic systems [11, 36, 42-44] have served as an alternative rehabilitation tool for stretching of the ankles and training for functional movement. Specifically, robot-aided rehabilitation of the ankle has been used effectively in treating ankle contracture and/or spasticity, and previous studies indicated that this intervention reduced ankle joint resistance torque and stiffness, increased ROM [10], and improved balance and gait $[34,35]$ in neurologically impaired patients. Robotic intervention in the form of intelligent stretching and active movement training has been studied in children with cerebral palsy and showed significant improvement in 12 children with CP in terms of improved passive and active ranges of motion, selective motor control, and mobility functions after 18 sessions of training (three sessions/week for 6 weeks) [12]. Waldman et al investigated the effects of the robotic rehabilitation of the ankle in stroke with ankle impairment, and the results showed subjects in the robot group improved significantly more in dorsiflexion PROM, dorsiflexion strength, and balance and walking function, while the spasticity measured by the Modified Ashworth Scale was reduced more than that in the control group [45]. Forrester et al [46, 47] conducted similar studies, and the results suggested that robotic rehabilitation of the ankle was effective in improving balance, motor function, and mobility poststroke. In this study, to forcefully and safely stretch the ankle of a subject with spasticity to its extreme positions, we used an intelligent stretching device that stretched the joint with quantitative feedback control of the resistance torque and stretching velocity. The stretching device was driven by a servomotor controlled by a digital signal processor (DSP) [18]. The exact rules of the DSP-controlled motion have been described elsewhere [10]. By using this control strategy, the stretching device moved quickly in the middle (non-spastic) ROM and slowed in the stiffer part of the ROM, while never exceeding preset stretching torques. This study demonstrated that improvement associated with the intelligent stretching of the spastic ankles post-stroke was consistent with previous research, and resulted in increased ROM and muscle strength, decreased ankle stiffness, and improved balance and mobility function. This type of high intensity, repetitive and efficient stretching can be more labor-saving and readily available to patients without the need of a skilled therapist. Furthermore, in this study, a significant correlation ( $\tau=0.265, P=0.041)$ was observed between MAS and DF stiffness, which is consistent with previous research. This measure of stiffness may potentially be used to obtain more accurate and quantitative evaluation of spastic hypertonia in future [11]. 


\section{Balance control}

Postural stability, often defined as balance, is the ability of the body to maintain its center of mass over the base of support [48], and it plays an important role in the recovery of motor function in patients with hemiplegia. Three kinds of strategy are involved in postural control in humans: ankle strategy, hip strategy, and stride strategy $[28,29]$. The ankle strategy refers to the body's center of gravity rotating or swinging around the ankle joint in a pendulum movement, which is the main strategy in normal people for maintaining balance when the support surface is firm and the perturbations are small [24, 49], while the hip strategy is mainly activated when the range of body sway is large. The most important roles of the ankle joints and the feet are in controlling body sway and forward movements of the lower extremities, and these roles require a sufficient range of motion, muscle strength, and proprioceptive sense in the ankle joints [50]. The ankle strategy is damaged partially after stroke creating muscular imbalance surrounding the ankle, increased joint stiffness, decreased proprioception of the ankle, and wrong central integration, which causes functional instability and/or imbalance [51].

At present, various therapeutic methods have been used to improve balance post-stroke, such as taskrelated training assisted-robot walking, virtual reality rehabilitation, core strength exercises [7], visual feedback training etc $[52,53]$. These methods of rehabilitation treat the patient as a whole, aiming to improve the posture control of the trunk and lower limbs. In our study, we focused on the reapplication of the ankle strategy to improve balance via robot-aided rehabilitation of the ankle. It forcefully, safely, and repeatedly stretched the ankle to its extreme positions resulting in structural changes in the viscoelastic properties of the connective tissues, thereby reducing ankle stiffness and increasing sensory inputs around the ankle joints (including cutaneous receptors, muscle-spindle receptors, and Golgi tendon organs located in the muscles, tendons, and ligaments). The patients were asked to stare at the display screen where an amplified and lateral "ankle joint" image was shown as stretching the ankle from dorsiflexion to plantarflexion, which was a real-time visual feedback integrating proprioception with the environment, promoting neuromuscular control during training $[54,55]$.

\section{Pro-Kin balance test}

To measure the ability to balance quantitatively, the Pro-Kin is a useful piece of equipment that has demonstrated reliability with the capability to feasibly generate instantaneous data regarding CoP, including the perimeter, ellipse, and movement in both the forward/backward and medial/lateral directions [31]. This balance test system only requires patients to have a certain ability to sit or stand, and it can find subtle balance differences more comprehensively, making up for the measurement errors caused by subjective factors in the balance scales assessment [56]. In this study, the Pro-Kin was used to quantitatively evaluate the standing static balance of patients before and after training, excluding the influences of the hip strategy and stride strategy, and explore the role of ankle strategy in balance more accurately $[57,58]$. The results showed that, after the intelligent stretching training of the ankle joint, trajectory lengths, elliptical trajectory, SD M/L, AS F/B with closed eyes, and SD F/B with opened eyes 
decreased significantly, but there were no significant decreases in the control group, which confirmed that robot-aided rehabilitation of the ankle is of great significance in the implementation of ankle strategy for the reestablishment of the correct depiction of the proprioceptive and muscular motor sensations. However, the balance scales failed to reflect the subtle differences in balance function after training between the two groups quantitatively. Quantitative results of the Pro-Kin balance test have the potential to increase the sensitivity of measures to guide treatment approaches and track patient progress over time.

Furthermore, we found that there were no significant improvements in trajectory lengths, elliptical trajectory, SD M/L, or AS M/L with opened eyes in the study group after training. As an explanation, we consider the following factors. First, on the stable support surface, the weight effects of proprioception, vestibule, and vision on the balance were $70 \%, 20 \%$, and $10 \%$, respectively [59], which showed that proprioception was the most important factor in maintaining balance when standing and walking on a flat surface. Visual compensation is extremely important when proprioception is destroyed post stroke [60]. However, it is more difficult to maintain balance while conducting the Pro-Kin test with eyes closed as the visual compensation is impaired, so significant improvements were easier to find with eyes closed but not open [61]. Secondly, when standing normally on a flat and solid surface, the limit of stability (LOS) was $8^{\circ}$ in the medial, lateral, forward directions and $4.5^{\circ}$ in the backward direction [62]. It was more difficult to maintain balance in the forward/backward direction, so the SD and AS of F/B may reflect the changes in balance more sensitively. In our study, the SD and AS of F/B deceased with open eyes after intelligent stretching training, which implied sensitive feed-back and feed-forward control mechanisms were improved [63].

\section{Correlations between DF stiffness and balance}

There has been no study that quantitatively analyzed the impact of local biomechanical properties of the ankle on the overall balance function. This study further explored the correlation between ankle stiffness and the Pro-Kin balance test outcomes with opened eyes. The findings showed that the stiffness of dorsiflexion was positively related to trajectory length, elliptical trajectory, and average velocity $M / L$ and F/B with opened eyes, and trajectory length was strongly positively related to the stiffness of dorsiflexion ( $=0.522, P=0.001)$, which meant that greater DF stiffness resulted in a worse balance function. But there was no significant correlation between PF stiffness and the Pro-Kin balance test outcomes, suggesting that DF stiffness was an important factor affecting the balance function, while PF stiffness was not, but the mechanism was not clear. We assumed that the decreased DF stiffness might activate the muscles around the ankles (especially the ankle dorsal flexor muscle) and increase the proprioceptive sense inputs and ROM of the ankle joint, so that the ability to appropriately control balance during sway was improved through better coordination and mobilization of the senses and muscle functions of the ankle after intelligent stretching. 


\section{Study limitations}

This study had some limitations. First, a small number of subjects were enrolled; further studies should increase the number of subjects to increase the power of the study. Second, no significant differences in walking function between the two kinds of intervention were found. This might be because the training frequency, intensity, and total repetitions were not optimal, or the long-term effects of intelligent stretching training were unknown, so further follow-up is necessary to explore the effects on walking function, and further studies should focus on exploring the optimal training protocol. Moreover, the patients in our study were adults with subacute stroke, and thus the results do not apply to patients with acute and chronic stroke. Hence, the possible effects of the intervention on patients with acute and chronic stroke require further investigation.

\section{Conclusions}

The robot-aided rehabilitation of the ankles provided well-controlled passive stretching to stroke survivors with ankle impairments, and benefits included improvements in biomechanics, spasticity, balance, motor function, and ADL post-stroke. Findings in this study suggested that robot-aided rehabilitation may be a beneficial addition to rehabilitation programs. As an important part of posture control, ankle strategy was of great significance for improving the overall balance. In particular, stiffness of dorsiflexion was an important factor affecting the balance. As a local biomechanical property of the ankle, it may be a sensitive indicator for evaluating the balance function after rehabilitation and predicting the risk of falls in the future.

\section{Abbreviations}

ROM: Range of Motion; CoP: Center of Pressure; MAS: Modified Ashworth Scale; DF dorsiflexion; PF: plantarflexion; FM-LE: Fugl-Meyer Motor Assessment of Lower Extremity; BBS: Berg Balance Scale; 6MWT: 6-minute walk test; PASS: Postural Assessment Scale for Stroke Patients; MBI: Modified Barthel Index; ADL: Activities of daily living; OE: Opened eyes; CE: Closed eyes; SD: Standard deviation; AS: Average speed; M/L: Medial/Lateral; F/B: Forward/Backward; BMI: Body Mass Index; M: male; F: female; DSP: Digital signal processor.

\section{Declarations}

\section{Acknowledgments}

We thank for Yupeng Ren PhD thoughtful suggestions, for Jiehua Yu assistance for biomechanical evaluations and calculations, Meizhen Huang PhD assistance for statistical analysis, and all the patients for participating in this experiment. We thank International Science Editing (http://www.internationalscienceediting.com) for editing this manuscript. 


\section{Authors' contributions}

YP and LZ contributed to the study design, analysis and interpretation of data and revisions to the manuscript. QW contributed to the analysis and interpretation of data. XZ contributed to the study design, data collection, analysis and interpretation of data and drafting the manuscript. Data collection was performed by $\mathrm{XZ}, \mathrm{XL}$ and $\mathrm{QX}$. YZ and SF performed experiments. All authors read and approved the manuscript submitted and agree to be accountable for all aspects of the work.

\section{Funding}

This study was supported by Beijing Municipal Natural Science Foundation (L182028) and Beijing Municipal Science and Technology Commission (Z181100009218003 and Z181100003118004). No additional external funding was received for this study. The funders had no role in study design, data collection and analysis, decision to publish, or preparation of the manuscript.

\section{Availability of data and materials}

The datasets used and analyzed during the current study are available from the corresponding author upon appropriate request.

\section{Ethics approval and consent to participate}

All protocols were reviewed and approved by the Beijing Tsinghua Chang Gung Hospital Medical Ethics (18172-0-01) according to the Declaration of Helsinki. Informed written consent was obtained from each subject in this study. This study is registered at http://www.chictr.org.cn under the study identifier ChiCTR2000030108.

\section{Consent for publication}

Consent for publication were included as part of informed consent.

\section{Competing interests}

Li-Qun Zhang holds an equity position in Beijing LTK Science and Technology Co., which made the ankle rehabilitation robot used in this study. The other authors declare that they have no competing interests. 


\section{Author details}

${ }^{1}$ Department of Rehabilitation, Beijing Tsinghua Changgung Hospital, Beijing, China. ${ }^{2}$ School of Clinical Medicine, Tsinghua University, Beijing, China. ${ }^{3}$ Departmennt of Physical Therapy \& Rehabilitation Science, University of Maryland, Baltimore, MD, USA. ${ }^{4}$ Departmennt of Orthopaedics, University of Maryland, Baltimore, MD, USA. ${ }^{5}$ Departmennt of Bioengineering, University of Maryland, College Park, MD, USA.

\section{References}

[1] Sun GC, Rey EV, Bai Z, Roth EJ, Zhang LQ. Biomechanic changes in passive properties of hemiplegic ankles with spastic hypertonia. Arch Phys Med Rehabil.2004;85:1646.

[2] Olney SJ, Richards C. Hemiparetic gait following stroke. Part l: Characteristics. Gait \& Posture 1996; 4:148.

[3] Lamontagne A, Malouin F, Richards CL. Contribution of passive stiffness to ankle plantarflexor moment during gait after stroke. Archives of Physical Medicine \& Rehabilitation.2000;81:351-58.

[4] Van der Linden M. Gait Analysis, Normal and Pathological Function, 2nd ed. J. Perry, J.M. Burnfield, Slack Inc., 576 pages, ISBN 978-1-55642r-r766-4. 2011. 97(2): p. 180-0.

[5] Thilmann AF, Fellows SJ, Ross HF. Biomechanical changes at the ankle joint after stroke. Journal of Neurology Neurosurgery \& Psychiatry.1991;54:134-39.

[6] Rymer WZ, Katz RT. Mechanisms of spastic hypertonia. Physical Medicine and Rehabilitation: State of the Art Reviews. 1994; 8:441-454.

[7] Bell KR, Vandenborne K. Contracture and limb deformities Principles of Neurologic Rehabilitation. In: Lazar RB, editors. New York: McGraw-Hill;1998. p. 309-328.

[8] Tabary JC, Tabary C, Tardieu C, Tardieu G, Goldspink G. Physiological and structural changes in the cats' soleus muscle due to immobilization at dierent lengths of plaster casts. Journal of Physiology. 1972;224: 231-244. 37.

[9] Williams PE, Goldspink G. Connective tissue change in immobilized muscle. Journal of Anatomy. 1984;138 (Pt 2):343-50.

[10] Zhang LQ, Chung SG, Bai Z, Xu D, ven Rey EMT, Rogers MW, Johnson ME and Roth EJ. Intelligent stretching of ankle joints with contracture/spasticity. IEEE Trans Neural Sys and Rehab Eng. 2002; 10:149-157. 
[11] Selles RW, Li X, Fang L, Sun GC, Roth EJ, Zhang LQ. Feedback-Controlled and Programmed Stretching of the Ankle Plantarflexors and Dorsiflexors in Stroke: Effects of a 4-Week Intervention Program. Arch Phys Med Rehabil.2005;86:2330-2336.

[12] Wu YN, Hwang M, Ren Y, Gaebler-Spira D, Zhang LQ. Combined Passive Stretching and Active Movement Rehabilitation of Lower-Limb Impairments in Children with Cerebral Palsy Using a Portable Robot. Neurorehabilitation \& Neural Repair. 2011; 25:378-85.

[13] Sukal-Moulton T, Clancy T, Zhang L, Gaebler-Spira D. Clinical Application of a Robotic Ankle Training Program for Cerebral Palsy Compared to the Research Laboratory Application: Does It Translate to Practice? Archives of Physical Medicine \& Rehabilitation. 2014; 95:1433-40.

[14] Forrester LW, Roy A, Krywonis A, Kehs G, Krebs HI, Macko RF. Modular Ankle Robotics Training in Early Subacute Stroke: A Randomized Controlled Pilot Study. Neurorehabilitation \& Neural Repair. 2014; 28:678-87.

[15] Lee Y, Chen K, Ren Y, Son J, Cohen BA, Sliwa JA, et al. Robot-guided ankle sensorimotor rehabilitation of patients with multiple sclerosis. Multiple Sclerosis \& Related Disorders. 2017; 11:65-70.

[16] Wikstrom EA, Tillman MD, Chmielewski TL, Borsa PA. Measurement and Evaluation of Dynamic Joint Stability of the Knee and Ankle After Injury. Sports Medicine. 2006; 36:393-410.

[17] Kligyte I, Lundyekman L, Medeiros JM. Relationship between lower extremity muscle strength and dynamic balance in people post-stroke. Medicina.2003;39: 122-128.

[18] Zhang LQ, Rymer WZ. Simultaneous and nonlinear identification of mechanical and reflex properties of human elbow joint muscles. IEEE Transactions on Biomedical Engineering. 1997; 44:1192-209.

[19] Zhang LQ, Wang G. Dynamic and static control of the human knee joint in abduction-adduction. Journal of Biomechanics. 2001; 34:1107-15.

[20] Olmstead TG, Wevers HW, Bryant JT and Gouw GJ. Effect of muscular activity on valgus/varus laxity and stiffness of the knee. Journal of Biomechanics. 1986;19(8), 565-577.

[21] Muraoka, T. Elastic properties of human Achilles tendon are correlated to muscle strength. Journal of Applied Physiology. 2005;99:665-69.

[22] Bohannon RW, Smith MB. Interrater reliability on a modified Ashworth scale of muscle spasticity. Phys Ther.1987;67:206-207.

[23] Fugl-Meyer AR, Jsk L, Leyman IL, Olsson S, Steglind S. The post stroke hemiplegic patient. I. A method for evaluation of physical performance. Scand J Rehabil Med. 1975; 7:13-31. 
[24] Berg K. Measuring balance in the elderly: validation of an instrument. Can J Public Health 1992; 83 Suppl 2: S7-11.

[25] Kaufman M, Moyer D, Norton J. The significant change for the Timed 25-Foot Walk in the Multiple Sclerosis Functional Composite. Multiple Sclerosis. 2000; 6:286-90.

[26] Benaim C, Perennou DA, Villy J, Rousseaux M, Pelissier JY. Validation of a standardized assessment of postural control in stroke patients: The postural assessment scale for stroke patients (PASS). Stroke.1999;30:1862-1868.

[27] Shah S, Vanclay F, Cooper B. Improving the sensitivity of the Barthel Index for stroke rehabilitation. Journal of Clinical Epidemiology.1989; 42:703-09.

[28] Shumway-Cook A, Woollacott M. Normal postural control. Motor control: Translating research into clinical practice. Philadelphia: Lippincott Williams and Wilkins. 2007:157-186.

[29] Winter DA. Human balance and posture control during standing and walking. Gait \& Posture.1995; 3(4),193-214.

[30] Srivastava A, Taly AB, Gupta A, et al. Post-stroke balance training: role of force platform with visual feedback. Neurol Sci.2009; 287:89-93.

[31] Xu HP, Quan LJ, Qiu Z, et al. Study on validity and reliability of PROKIN balance instrument for predicting fall in elderly people. Nanchang Univ (Med Sci). 2012; 52:34-7.

[32] Field AP. Discovering statistics using SPSS for Windows: advanced techniques for the beginner. London: Sage; 2000.

[33] Bressel E, McNair PJ. The effect of prolonged static and cyclic stretching on ankle joint stiffness, torque relaxation, and gait in people with stroke. Phys Ther.2002;82:880-887.

[34] Yeh CY, Chen JJJ, Tsai KH. Quantitative analysis of ankle hypertonia after prolonged stretch in subjects with stroke. Journal of Neuroscience Methods. 2004; 137:305-14.

[35] Mcnair PJ, Dombroski EW, Hewson DJ, Stanley SN. Stretching at the ankle joint: viscoelastic responses to holds and continuous passive motion. Med Sci Sports Exerc.2001;33:354-58.

[36] Lippincott Williams, Wilkins. Correction to: Guidelines for Adult Stroke Rehabilitation and Recovery: A Guideline for Healthcare Professionals From the American Heart Association/American Stroke Association. Stroke. 2017;48(2): e78.

[37] Chen $\mathrm{CH}$. Effect on spasticity after performance of dynamic-repeated-passive ankle joint motion exercise in chronic stroke patients. Kaohsiung Journal of Medical Sciences. 2006; 22:610-17. 
[38] Thibaut A, Chatelle C, Ziegler E, Bruno MA, Laureys S, Gosseries O. Spasticity after stroke: Physiology, assessment and treatment. Brain Injury. 2013; 27:1093-1105.

[39] Gandolfi M. Rehabilitation procedures in the management of spasticity. Eur J Phys Rehabil Med. 2010; 46:423-38.

[40] Abo M. Effect of home-based training using a slant board with dorsiflexed ankles on walking function in post-stroke hemiparetic patients. Journal of Physical Therapy Science. 2016; 28:2353-57.

[41] Choi JD. The effect of balance training with plantar flexor stretching on range of motion, balance, and gait in stroke patients: a randomized controlled pilot trial. Phys. Therapy Rehabil Sci. 2015;4: 66-72.

[42] Zhang LQ. Developing a Wearable Ankle Rehabilitation Robotic Device for in-Bed Acute Stroke Rehabilitation. IEEE Trans Neural Syst Rehabil Eng. 2017; 25:589-96.

[43] Charrotton Y. Improvement of lower limb functioning in ambulatory chronicle stroke patients undergoing robot-assisted training. Annals of Physical \& Rehabilitation Medicine. 2016;59:e89.

[44] Gao F, Ren Y, Roth EJ, Harvey R, Zhang and LQ. Effects of repeated ankle stretching on calf muscletendon and ankle biomechanical properties in stroke survivors. Clinical Biomechanics. 2011; 26: 516522.

[45] Waldman G, Yang CY, Ren Y, Liu L, Zhang LQ. Effects of robot-guided passive stretching and active movement training of ankle and mobility impairments in stroke. Neurorehabilitation. 2013; 32:625-34.

[46] Forrester LW, Roy A, Goodman RN, et al. Clinical application of a modular ankle robot for stroke rehabilitation. NeuroRehabilitation.2013; 33(1): 85-97.

[47] Forrester LW, Roy A, Krywonis A, Kehs G, Krebs HI, Macko RF. Modular Ankle Robotics Training in Early Subacute Stroke: A Randomized Controlled Pilot Study. Neurorehabilitation \& Neural Repair. 2014; 28:678-87.

[48] Shumway-Cook A, Woollacott M. Motor Control. Translating Research into Clinical Practice. 3rd ed. Baltimore: Lippincott Williams \& Wilkins, 2007.

[49] Kanekar N, Aruin AS. Aging and balance control in response to external perturbations: role of anticipatory and compensatory postural mechanisms. Age. 2014, 36(3):517-521.

[50] Neumann DA: Kinesiology of the Musculoskeletal System: Foundations for Physical Rehabilitation. Mosby, 2002; 528-529.

[51] Lieber RL, Bodine-Fowler SC. Skeletal muscle mechanics: implications for rehabilitation. Phys Ther.1993;73:844-56. 
[52] Lloréns R, Noé E, Colomer C, Alca Iz M. Effectiveness, Usability, and Cost-Benefit of a Virtual RealityBased Telerehabilitation Program for Balance Recovery After Stroke: A Randomized Controlled Trial. Arch Phys Med Rehabil.2015;96(8):1544.

[53] Kim KH, Lee KB, Bae YH, Fong SSM, Lee SM. Effects of progressive backward body weight supported treadmill training on gait ability in chronic stroke patients: A randomized controlled trial. Technology \& Health Care Official Journal of the European Society for Engineering \& Medicine.2017;25:1-10.

[54] Prentice WE. Balance and Joint Stability: The Relative Contributions of Proprioception and Muscular Strength. Journal of Sport Rehabilitation. 2000; 9:315-28.

[55] Fu FH. The Role of Proprioception in the Management and Rehabilitation of Athletic Injuries. American Journal of Sports Medicine. 1997; 25:130-37.

[56] Mauch M, Kalin X. Reliability of the Prokin Type B Line System (TechnoBody ${ }^{T M}$ ) Balance System. Internal Project Report. Praxisklinik Rennbahn. 2011; 1-9.

[57] Srivastava A, Taly AB, Gupta A, et al. Post-stroke balance training: role of force platform with visual feedback. Neurol Sci. 2009;287: 89-93.

[58] Di X, Li X, Zhai Y, Neurology DO, Xian GH. Effects of Pro-Kin balance training on balance and the risk of fall in stroke patients. Chin J Rehabil. 2017; 32:196-8.

[59] Peterka Robert J, Loughlin Patrick J, Dynamic regulation of sensorimotor integration in human postural control. Journal of Neurophysiology.2004; 91: 410-23.

[60] Nima T, Jane M, Armstrong D G, et al. The Influence of Diabetic Peripheral Neuropathy on Local Postural Muscle and Central Sensory Feedback Balance Control. Plos One. 2015; 10(8): e0135255-.

[61] Mulder T and Hulstyn W. Sensory feedback therapy and theoretical knowledge of motor control and learning. Am J Phys Med. 1984;63: 226-244.

[62] Duncan PW, Weiner DK, Chandler J, Studenski S. Functional reach: A new clinical measure of balance. J Gerontol.1990; 45: 192-197.

[63] Kim KS. The effects of ankle joint muscle strengthening and proprioceptive exercise programs accompanied by functional electrical stimulation on stroke patients' balance. Journal of Physical Therapy Science. 2015; 27:2971-75.

\section{Figures}




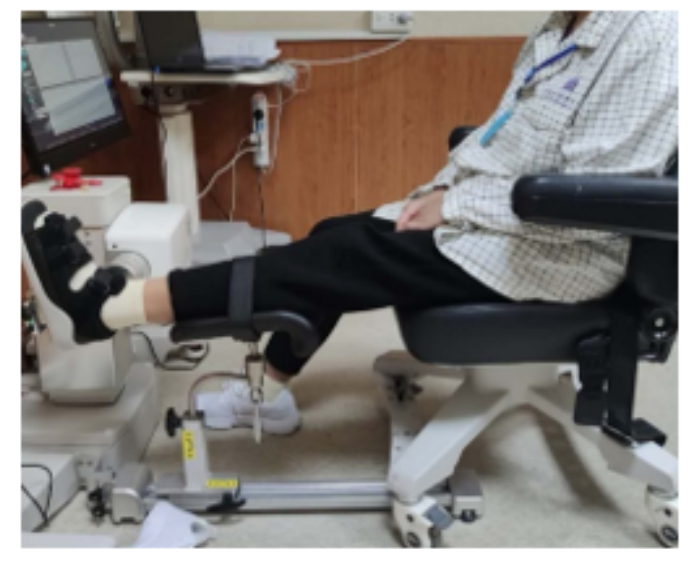

Figure 1

A patient seated in the ankle rehabilitation robot device.

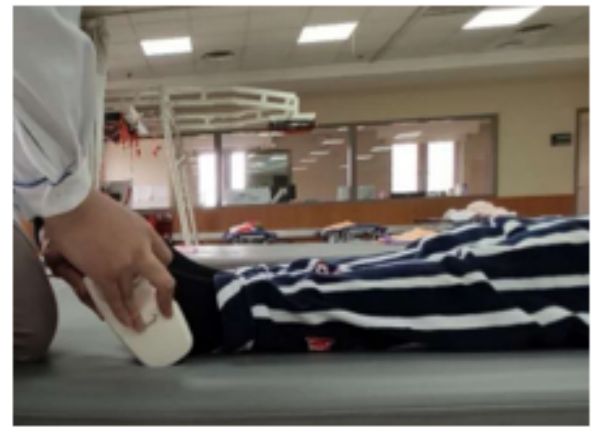

(a)

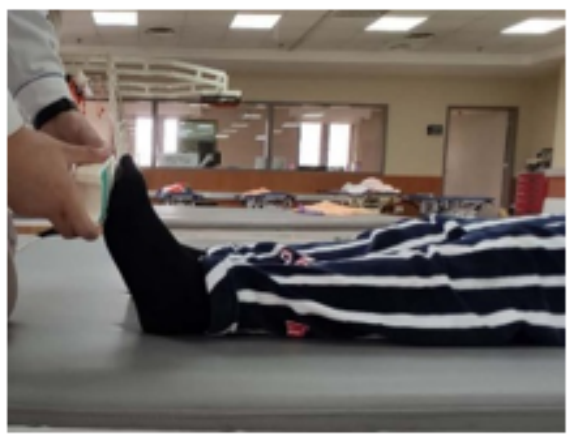

(b)

Figure 2

ROM (a) and muscle strength (b) measured by Hoggan MicroFET3 portable device.

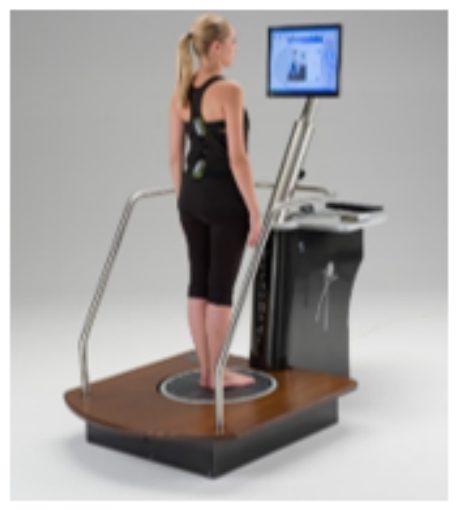

Figure 3

Static Balance Assessed by the Pro-Kin System 


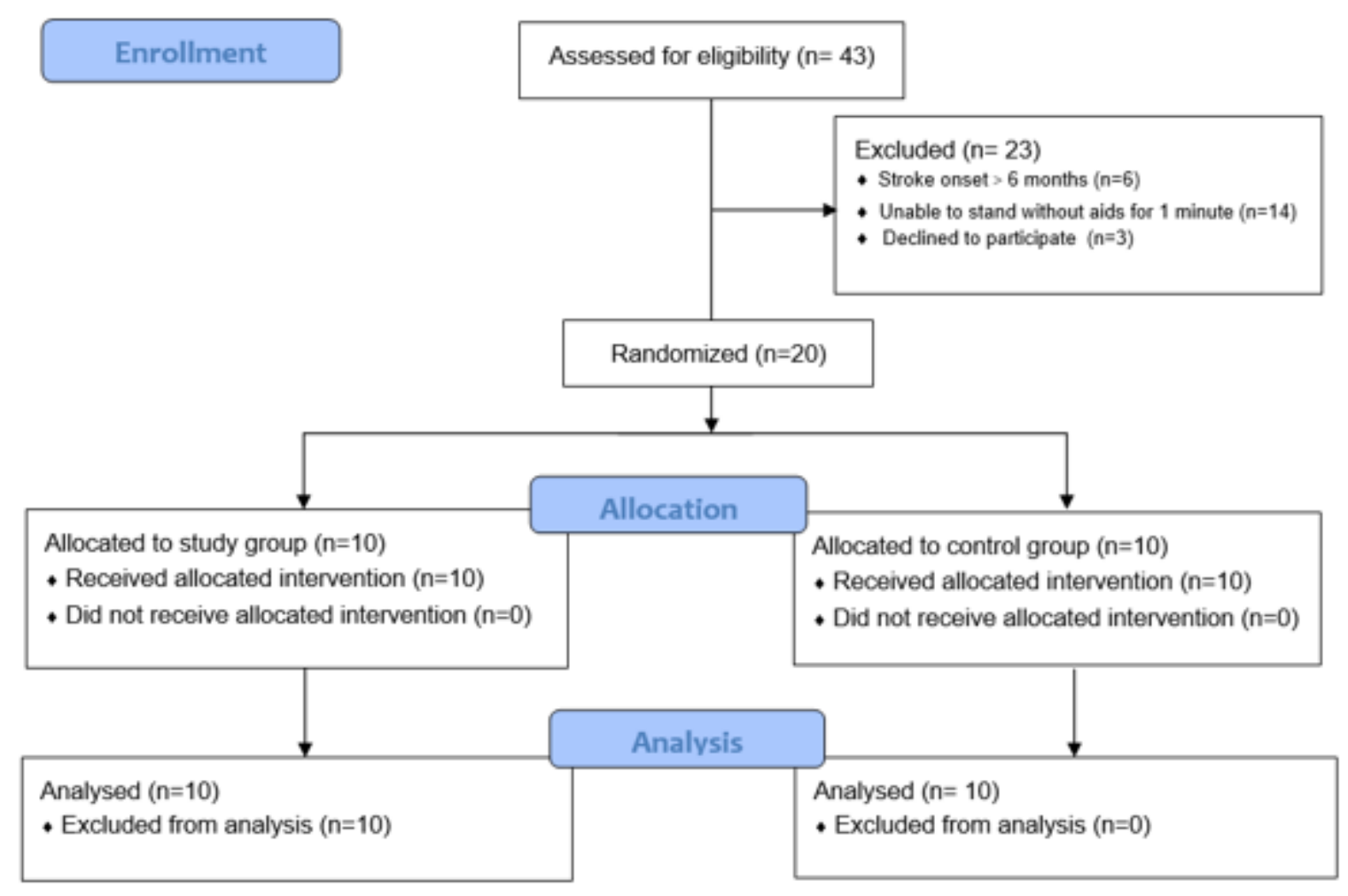

Figure 4

CONSORT patient flow throughout the study.

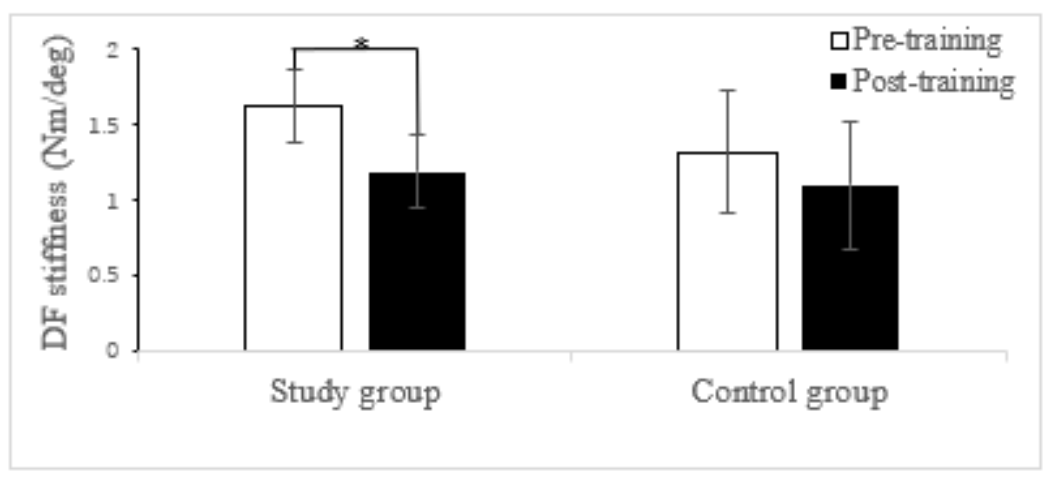

Figure 5

DF stiffness before and after 2 weeks of training in the two groups. ${ }^{*} \mathrm{P}<.05$.

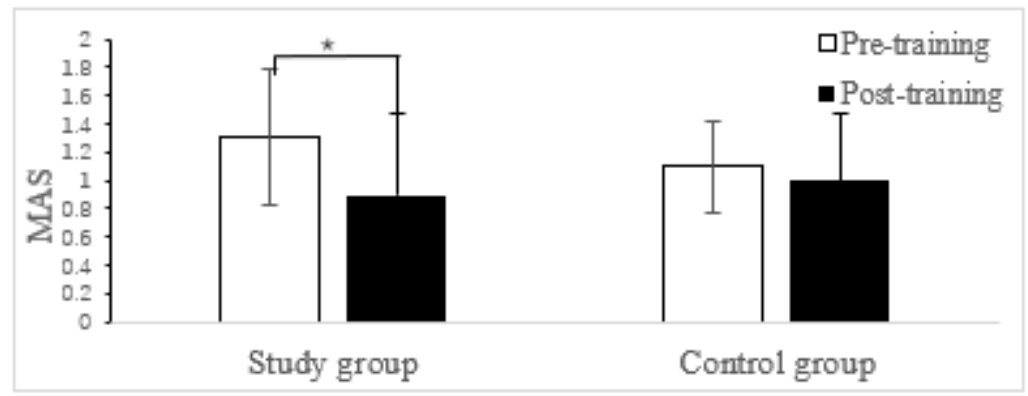


Figure 6

MAS before and after 2 weeks of training in the two groups. ${ }^{*}<<.05$.

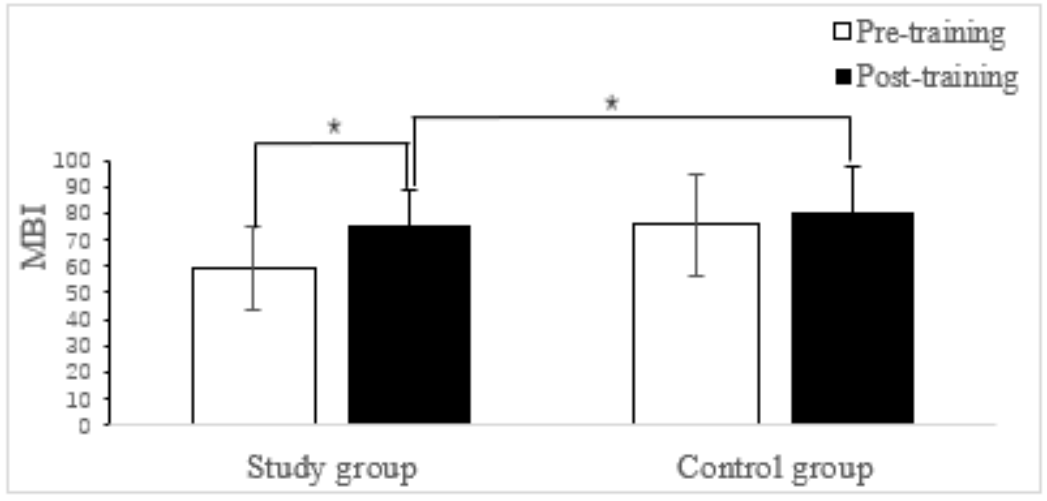

Figure 7

MBI before and after 2 weeks of training in the two groups. ${ }^{*}<<.05$.

Subject S09-Study Group

A. Trajectory of the CoP Pre-training (CF)

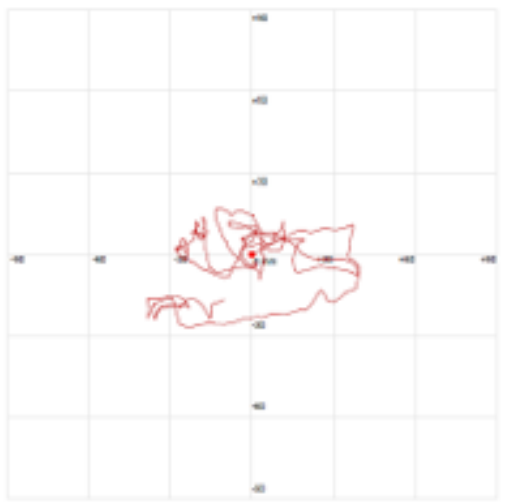

C. Trajectory of the CoP Pre-training (OF)

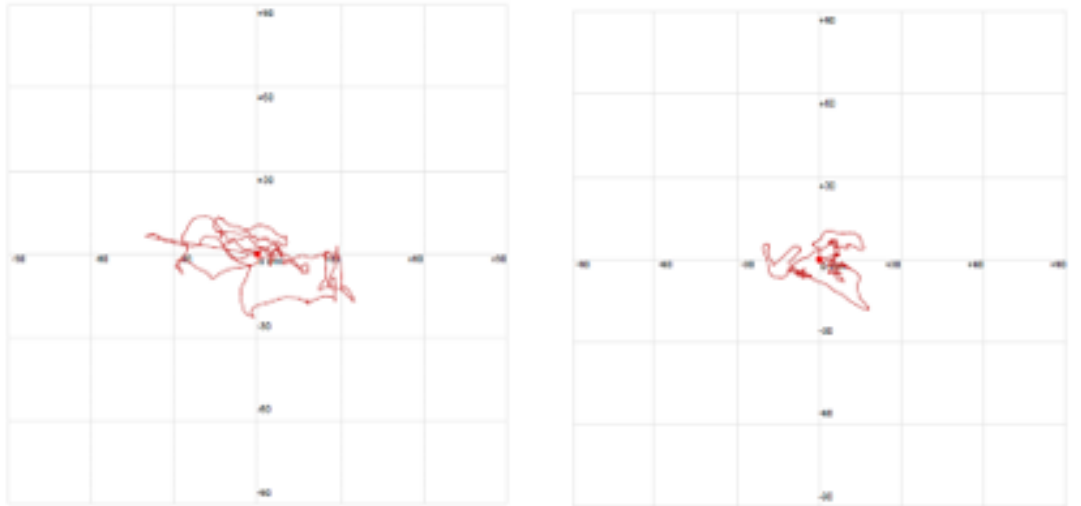

B. Trajectory of the CoP Post-training (CE)
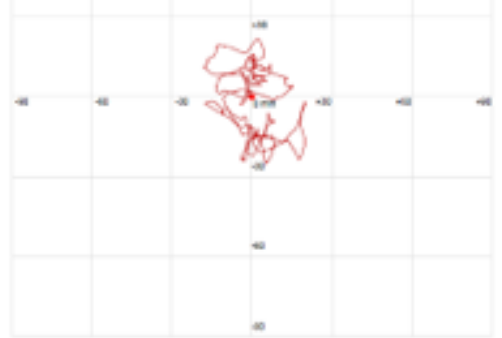

D. Trajectory of the CoP Post-training (OE)

\section{Figure 8}

Example of the trajectory of the center of pressure (CoP) of Pre-training $(a, c)$ and Post-training $(b, d)$ from the Study Group with opened eyes (OE) and closed eyes (CE). 
A. Trajectory of the CoP Pre-training (CF)

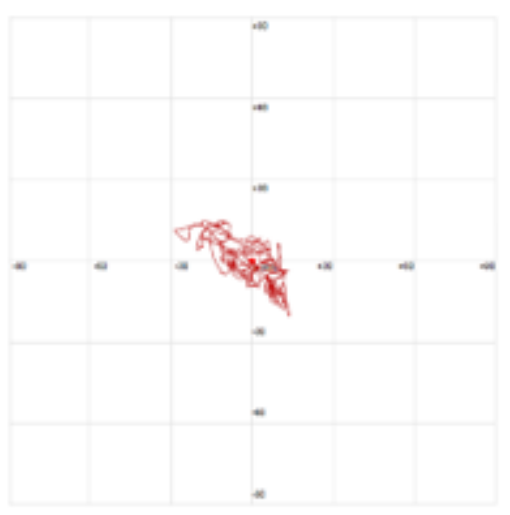

C. Trajectory of the CoP Pre-training (OF)

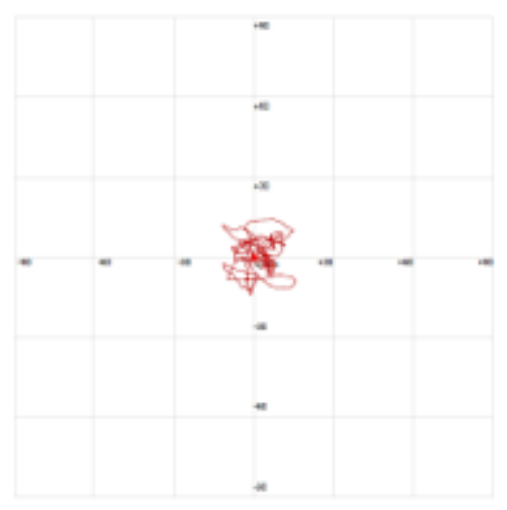

B. Trajectory of the CoP Post-training (CE)

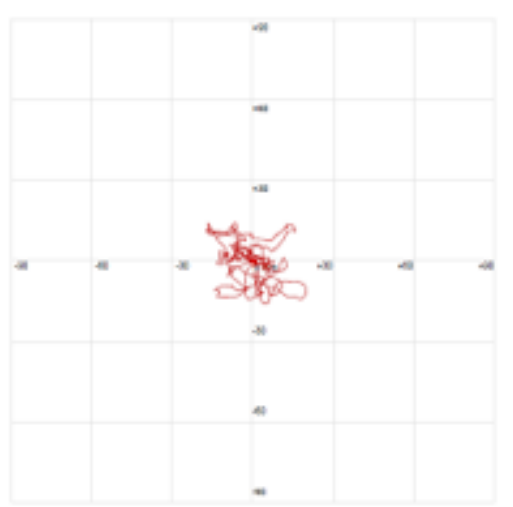

D. Trajectory of the CoP Post-training (OE)

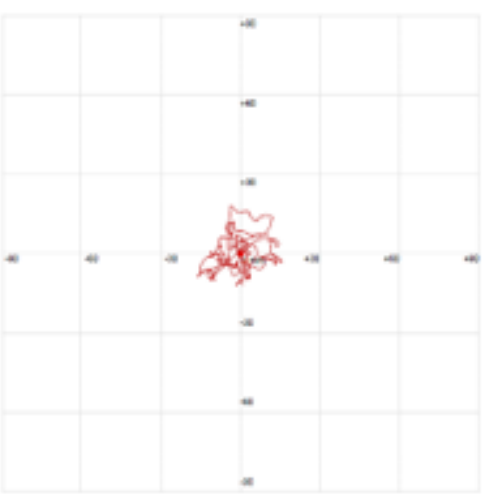

\section{Figure 9}

Example of the trajectory of the center of pressure (CoP) of Pre-training (a, c) and Post-training (b, d) from the Control Group with opened eyes (OE) and closed eyes (CE).

\section{Supplementary Files}

This is a list of supplementary files associated with this preprint. Click to download.

- CONSORT2010Checklist.doc 